\title{
Fat-forming Solitary Fibrous Tumor of the Kidney - A Case Report and Literature Review
}

\author{
NICOLAE BACALBASA ${ }^{1,2}$, IRINA BALESCU ${ }^{3}$, GEORGE JINESCU $^{4}$, \\ MADALINA MARCU ${ }^{5}$, ANDREEA CONTOLENCO ${ }^{5}$, DOINA POP ${ }^{6}$, \\ DRAGOS DOBRITOIU ${ }^{1,2}$, OLIVIA IONESCU ${ }^{7}$, PARIS IONESCU ${ }^{8}$ and CLAUDIA STOICA ${ }^{9}$ \\ ${ }^{1}$ Department of Obstetrics and Gynecology, \\ Carol Davila University of Medicine and Pharmacy, Bucharest, Romania; \\ ${ }^{2}$ Department of Obstetrics and Gynecology, Dr. Ioan Cantacuzino Clinical Hospital, Bucharest, Romania; \\ ${ }^{3}$ Department of General Surgery, Ponderas Academic Hospital, Bucharest, Romania; \\ ${ }^{4}$ Department of General Surgery, Floreasca Emergency Clinic Hospital, Bucharest, Romania; \\ Departments of ${ }^{5}$ Pathology and ${ }^{9}$ General Surgery, Ilfov Clinical County Hospital, Bucharest, Romania; \\ ${ }^{6}$ Department of Pathology Victor Babes National Institute of Pathology, Bucharest, Romania; \\ ${ }^{7}$ Department of Obstetrics and Gynecology, Fichtelgebirge Hospital, Marktredwitz, Germany; \\ ${ }^{8}$ Department of Obstetrics and Gynecology, Ovidius University of Medicine, Constanta, Romania
}

\begin{abstract}
Fat-forming solitary fibrous tumors arising from the kidney are extremely rare, with only few cases reported so far. We report the case of a 49-year-old patient investigated for lumbar pain and diagnosed preoperatively with a large retroperitoneal tumor destroying the right kidney. The patient was submitted to surgery, the tumor being resected en bloc with right nephrectomy. The histopathological studies demonstrated the presence of a hemangiopericytoma-like tumor with adipocytary component; the immunohistochemical studies demonstrated the presence of cluster of differentiation 34 (CD34), while human melanoma black (HMB45) staining was negative. Therefore, the diagnostic of fat-forming solitary fibrous tumor of the kidney was established. The postoperative outcome was uneventful, the patient being discharged on the sixth postoperative day. In conclusion, although fat-forming solitary fibrous tumor of the kidney is a very rare entity, this diagnosis should be taken into consideration whenever a large retroperitoneal mass with high adipose content and renal invasion is described at the preoperative studies.
\end{abstract}

Solitary fibrous tumors (SFT) were reported for the first time by Wagner in Leipzig, in 1870 (1). Initially described in the

This article is freely accessible online.

Correspondence to: Irina Balescu, Department of General Surgery "Ponderas" Academic Hospital, Bucharest, Romania. Tel: +40 724077709, e-mail: irina.balescu@ ponderas-ah.ro

Key Words: Fat-forming solitary fibrous tumor, kidney, nephrectomy. pleura, they were reported later in almost any extrapleural site (2, 3). Fat-forming SFT is a recently recognized type of SFT that usually arises in the deep soft tissues at the level of the lower extremities, as well as in the retroperitoneal area (4). This histopathological subtype was previously named lipomatous hemangiopericytoma and usually affects middleaged adults. When it comes to fat-forming SFT of the kidney, only few cases have been reported (5-7). In the current article, we present the case of a 49-year-old patient who was submitted to surgery for a large retroperitoneal tumor destroying the right kidney and in whom the histopathological and immunohistochemical studies confirmed this tumoral subtype.

\section{Case Report}

A 49-year-old obese patient with no prior surgical history presented for diffuse lumbar pain. The abdominal ultrasound described the presence of a large retroperitoneal tumor entirely destroying the right kidney, therefore the patient was submitted to computed tomography. The imaging studies confirmed the presence of a $15 \times 15 \times 10 \mathrm{~cm}$ large tumor involving the right kidney, in the absence of other lesions. The patient was submitted to surgery: intraoperatively a large encapsulated lesion destroying the right kidney was found; the tumor was resected en bloc with right nephrectomy (Figures 1-3). The postoperative course was uneventful, the patient being discharged on the sixth postoperative day. The histopathological studies revealed the presence of a $16 \times 14 \times 8 \mathrm{~cm}$ lesion with fatty aspect when sectioned. The tumor was well encapsulated 


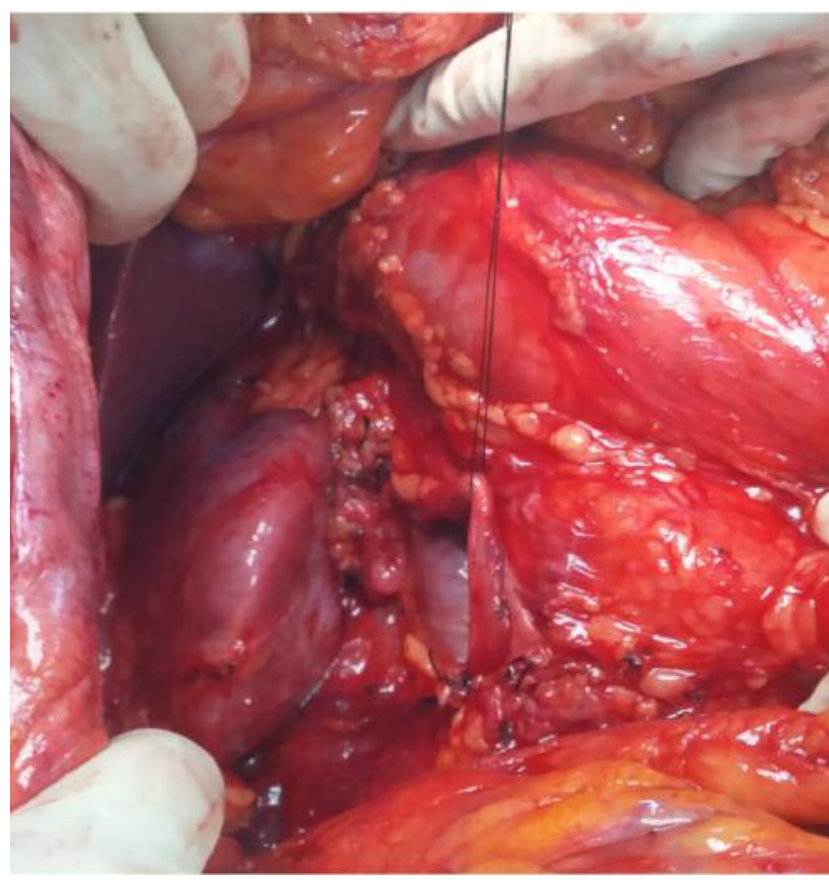

Figure 1. Large tumor deforming the right kidney. The right ureter was dissected and mobilized.

and presented both lesions of adipose tissue and hemorrhagic areas. The microscopic examination revealed the presence of an well-circumscribed mass with a fibrous capsule delimitating large areas of hemangiopericytomalike tissues as well as an adipose component (Figures 4 and 5). Diagnosis was of a fat-forming SFT of the tissue; however, immunohistochemical studies were requested in order to confirm the diagnosis. Immunohistochemical staining was positive for the multigenic family of calciummodulated proteins of the EF-hand type serum S100 (S100) in the adipose component, while cluster of differentiation 34 (CD34), desmin and smooth muscle actin (SMA) were positive at the level of the vascular and perivascular cells. Staining of CD99 and B-cell lymphoma 2 (BCL2) were diffusely positive in the lesion while those for melan A, calponin and human melanoma black 45 (HBM45) were negative. Moreover, Ki67 staining was positive in only up to $3 \%$ of the tissues. These findings established the positive diagnosis of fat-forming SFT of the kidney.

\section{Discussion}

Fat-forming SFT usually arises in middle-aged adult males and are most often found in the lower extremities, as well as in the retroperitoneal areas (4); less common localizations include the orbit, perineum mediastinum or spine (8-10). The histopathological studies of these tumors usually reveal a

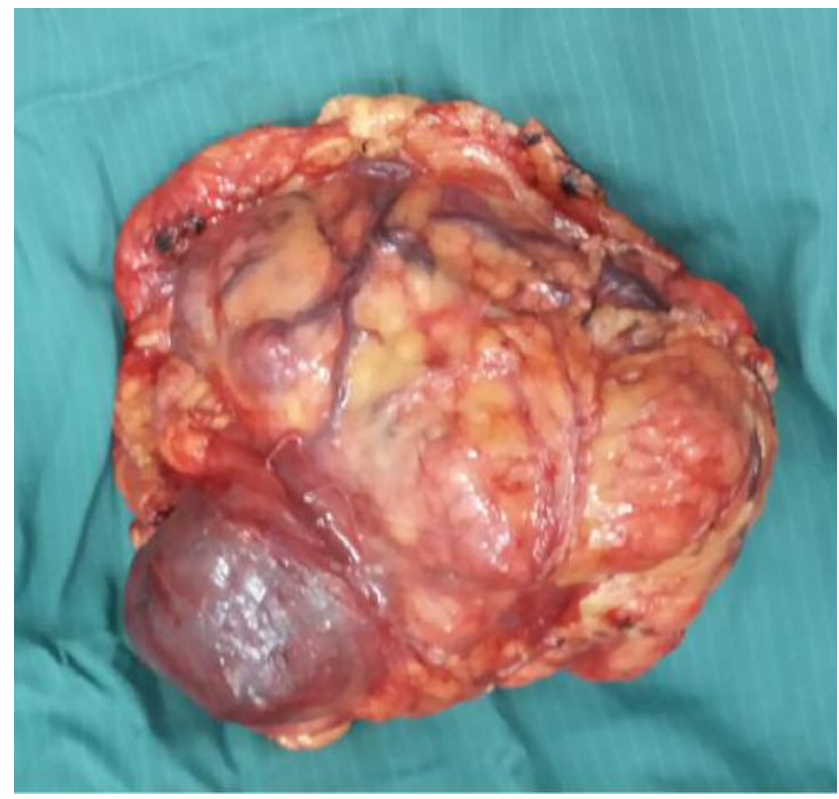

Figure 2. The aspect of the specimen - the right kidney was entirely deformed by the tumor measuring $16 \times 14 \times 8 \mathrm{~cm}$.

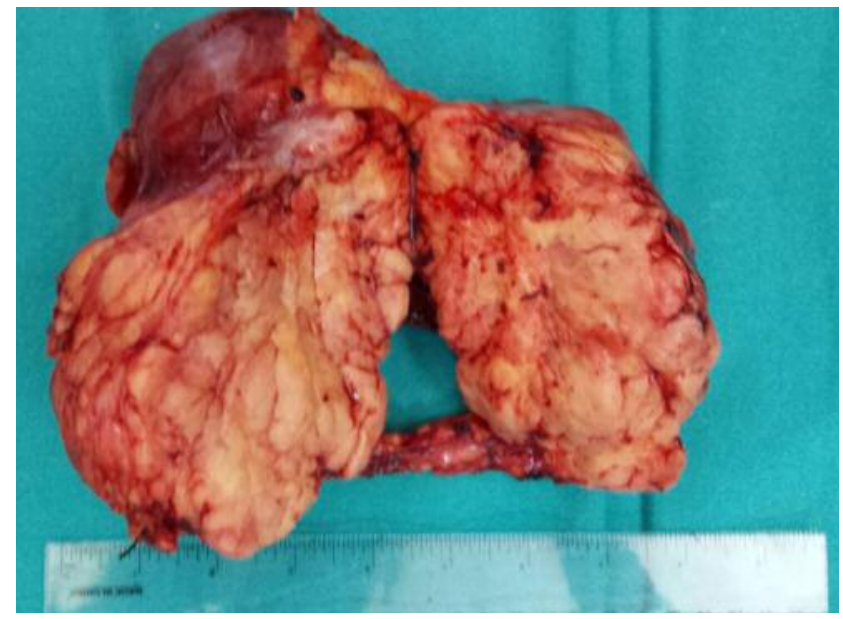

Figure 3. The specimen was sectioned - large areas of adipose tissues and hemorrhagic areas were visible.

non-adipose component with positive staining for CD34 and BCL2 in association with an adipose component composed mostly of benign adipocytes and atypical lipomatous tumorlike areas, closely mimicking a well-differentiated liposarcoma (11-12). When it comes to the origin of the adipocytic component, certain studies incriminated the pericyte cells $(12,13)$. 


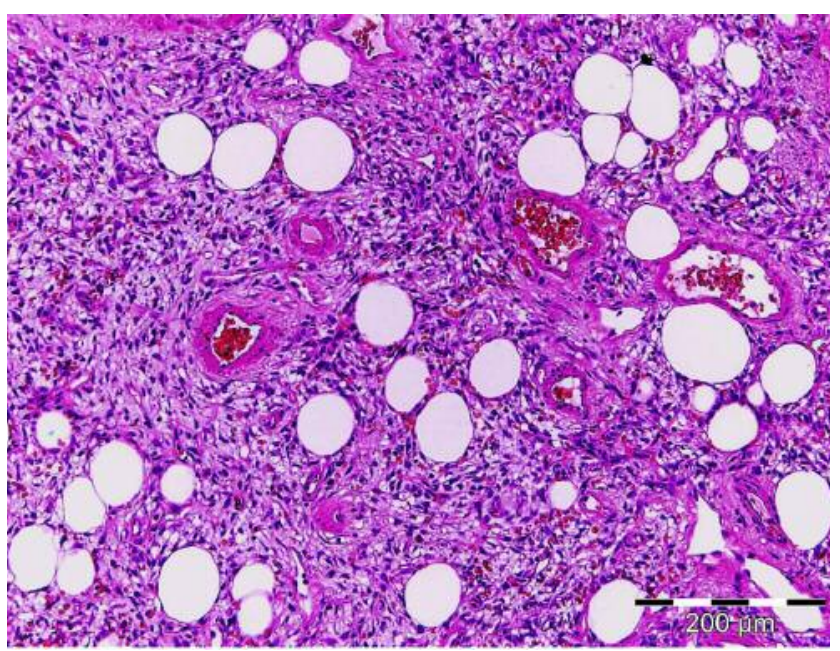

Figure 4. Mesenchymal proliferation with patternless architecture, showing intimate admixure of monomorphic spindle cells and mature adipocytes, and hemangiopericytoma-like branching vessels (magnification $\times 100)$.

As for the renal structure which might induce a fatforming SFT development, it has been demonstrated that the renal capsule as well as the pelvis and the adjacent pelvic areas are the most frequent origins; in the meantime, kidney invasion is rarely encountered (7).

During the preoperative evaluation, the aspect revealed by computed tomography might also suggest a diagnosis of a renal angiomyolipoma; however, histopathological studies demonstrating the presence of CD34 as well as the absence of HBM45 will lead to the final diagnosis of fat-forming SFT (7).

When it comes to the long-term prognosis of these cases, recurrences are rarely encountered, these tumors usually having a benign course. The low biological aggressiveness was also demonstrated in our case, the Ki67 index being positive in only $3 \%$ of all tumoral cells.

The first case of a fat-forming SFT was reported in 2005 by Yamaguchi et al. It was the case of a 51-year-old woman diagnosed with a large renal tumor arising from the renal capsule. The histopathological studies described the presence of a SFT; however, attention was focused on the presence of focal aggregates of fat cells which led to the diagnosis of a fat-forming SFT. Moreover, the authors underlined the necessity for establishing differential diagnostic of this histopathological entity from spindle cell carcinoma, gastrointestinal stromal tumor, angiomyolipoma or cellular schwannoma (6).

The second case of a fat-forming SFT of the tumor was published in 2014 by Cortes et al. it was the case of a 64year-old male patient who presented for epigastric pain and was diagnosed with a renal tumor; initially the tumor was

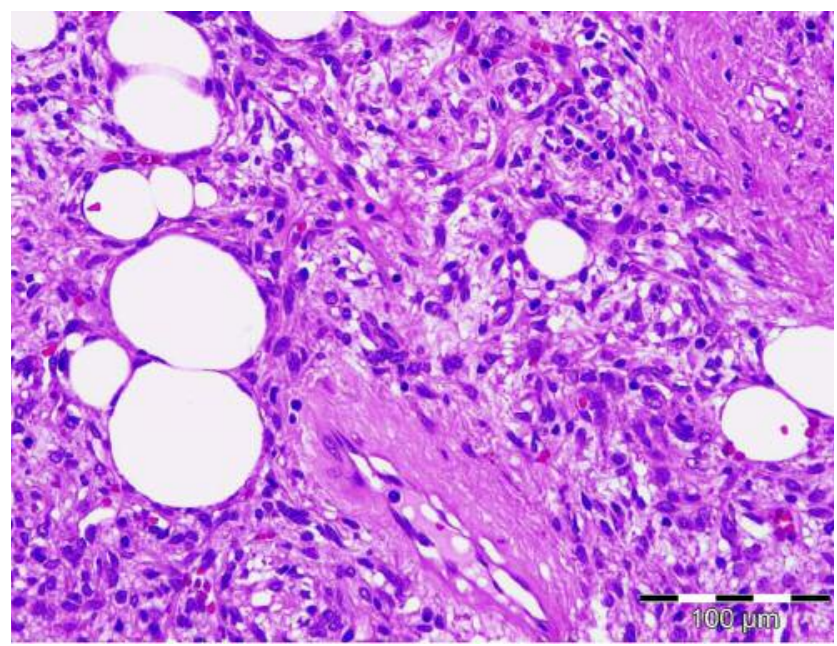

Figure 5. Typically bland ovoid to spindle-shaped cells, with pale cytoplasm, indistinct borders, admixed with mature adipocytes (magnification $\times 200$ ).

thought to be an angiomyolipoma. However, histopathological studies demonstrated the presence of a fat-forming SFT of the kidney (5).

Another similar case of fat-forming SFT of the kidney was reported by Chen et al. in 2015. In this article, the authors described the case of a 30-year-old woman who presented for right lower back pain and who was diagnosed at preoperative imagistic studies with a $60 / 45 \mathrm{~mm}$ renal tumor which had developed in the hilar area of the right kidney. The tumor was resected, the histopathological studies demonstrating the presence of a well-encapsulated tumor composed of hypocellular and hypercellular areas and lobules of mature adipose tissue. Immunohistochemical staining revealed a diffuse positivity for CD34 and BCL2 and negativity for HBM45, melan-A and SMA. Ki67 expression was present in fewer than $2 \%$ of all tumoral cells. All these pathological studies confirmed the diagnosis of a fat-forming SFT with low malignancy; at 19 months, the patient presented no sign of recurrent disease (7).

In order to establish the immunohistochemical modifications, which can predict the malignant transformation of SFT, Schulz et al. conducted a study on 294 patients with various types of SFT. Among these cases, the authors reported 223 cases of fibrous variant of the tumor and 65 cases presenting the cellular type, four of these cases being diagnosed as fat-forming SFT. Regarding the demographic characteristics of patients diagnosed with fat-forming SFT, there were two males and two females, three out of the four cases being younger than 60 years. In all cases, the tumor was smaller than $10 \mathrm{~cm}$ and was located in the lower extremities in two cases, in the abdomen in one and in the retroperitoneal area in one case. The immunohistochemical 
staining of this histopathological subtype revealed a high CD34 expression in two out of the four cases, a low mitotic index $(\mathrm{Ki} 67<5 \%)$ in three out of the four cases, a high level of BCL2in three out of the four cases and a high level of insulinlike growth factor 2 (IGF2) in all four cases. When analyzing the presence of malignancy criteria (consisting of the presence of distant metastases, necrosis, tumor size larger than $10 \mathrm{~cm}$ or $\mathrm{Ki} 67>20 \%$ ), the authors reported metastatic disease in 10 cases, necrosis in 10, large tumors in 39 and high Ki67 index in eight cases. As for the histopathological variant, the study concluded that $21 \%$ of the fibrous variant and $34 \%$ of the cellular variant were associated with at least one malignant feature. When analyzing the correlation between immunohistochemical staining and the presence of malignant features, the authors demonstrated that a reduced expression of CD34 and a high expression of IGF2 are strongly associated with a poorer outcome, as well as with the presence of at least one malignancy criterion (13).

When it comes to our case, according to the study by Schulz et al., two poor prognostic factors were associated: the tumor was larger than $10 \mathrm{~cm}$ and presented necrotic areas. However, its encapsulated character in association with the low mitotic index $(\mathrm{Ki} 67<3 \%)$ and the high expression of CD34 should offer a potential benefit for the long-term prognosis. Moreover, the complete resection of the tumor en bloc with the entire surrounding adipose tissue should offer a supplemental benefit.

\section{Conclusion}

Fat-forming SFT of the kidney is an extremely rare pathology, only few cases being reported. Although most cases have a favorable prognosis, recurrences being extremely rare, patients presenting lesions larger than $10 \mathrm{~cm}$, with high mitotic index, necrotic areas and negative staining for CD34 seem to be at risk of having a poorer outcome.

\section{References}

1 Wagner E: Das tuberkelähnliche Lymphadenom. In: Wagner E, Wunderlich C and Roser W (Eds.). Archiv der Heilkunde, Wigand, Leipzig, 1870.

2 Mosquera JM and Fletcher CD: Expanding the spectrum of malignant progression in solitary fibrous tumors: a study of 8 cases with a discrete anaplastic component - Is this dedifferentiated SFT? Am J Surg Pathol 33: 1314-1321, 2009.
3 Vallat-Decouvelaere AV, Dry SM and Fletcher CD: Atypical and malignant solitary fibrous tumors in extrathoracic locations: evidence of their comparability to intra-thoracic tumors. Am J Surg Pathol 22: 1501-1511, 1998.

4 Guillou L, Gebhard S and Coindre JM: Lipomatous hemangiopericytoma: a fat-containing variant of solitary fibrous tumor? Clinicopathologic, immunohistochemical, and ultrastructural analysis of a series in favor of a unifying concept. Hum Pathol 31: 1108-1115, 2000.

5 Cortes LG, Caserta NM and Billis A: Fat-forming solitary fibrous tumor of the kidney: a case report. Anal Quant Cytopathol Histpathol 36: 295-298, 2014.

6 Yamaguchi T, Takimoto T, Yamashita T, Kitahara S, Omura M and Ueda Y: Fat-containing variant of solitary fibrous tumor (lipomatous hemangiopericytoma) arising on surface of kidney. Urology 65: 175, 2005.

7 Chen Y, Wang F and Han A: Fat-forming solitary fibrous tumor of the kidney: a case report and literature review. Int J Clin Exp Pathol 8: 8632-8635, 2015.

8 Aftab S, Casey A, Tirabosco R, Kabir SR and Saifuddin A: Fatforming solitary fibrous tumour (lipomatous haemangiopericytoma) of the spine: case report and literature review. Skeletal Radiol 39: 1039-1042, 2010.

9 Pitchamuthu H, Gonzalez P, Kyle P and Roberts F: Fat-forming variant of solitary fibrous tumour of the orbit: the entity previously known as lipomatous haemangiopericytoma. Eye (Lond) 23: 1479-1481, 2009.

10 Kim MY, Rha SE, Oh SN, Lee YJ, Byun JY, Jung CK and Kang WK: Case report. Lipomatous haemangiopericytoma (fatforming solitary fibrous tumour) involving the perineum: CT and MRI findings and pathological correlation. Br J Radiol 82: e23e26, 2009.

11 Lee JC and Fletcher CD: Malignant fat-forming solitary fibrous tumor (so-called "lipomatous hemangiopericytoma"): clinicopathologic analysis of 14 cases. Am J Surg Pathol 35: 1177-1185, 2011.

12 Ceballos KM, Munk PL, Masri BA and O'Connell JX: Lipomatous hemangiopericytoma: a morphologically distinct soft-tissue tumor. Arch Pathol Lab Med 123: 941-945, 1999.

13 Schulz B, Altendorf-Hofmann A, Kirchner T, Katenkamp D, Petersen I and Knosel T: Loss of CD34 and high IGF2 are associated with malignant transformation in solitary fibrous tumors. Pathol Res Pract 210: 92-97, 2014. 